\title{
Revascularización miocárdica de la arteria descendente anterior con arteria mamaria interna izquierda con circulación extracorpórea: seguimiento a 10 años
}

\author{
Juan Carlos Bahamondes S, G ustavo Meriño S,
} Abelardo Silva von E, Juan Salman A.
Myocardial revascularization of the anterior descending coronary artery with left internal mammary artery by means of extracorporeal circulation: 10 years follow-up

Background: The use of left internal mammary artery (LMA) as a graft to anterior descending artery (LAD) has been associated with better long term results in coronary surgery. Aim: To assess and report the long-term results of LMA to LAD bypass grafting for isolated LDA lesions. Patients and methods: Retrospective analysis of the medical records and surgical protocols of 40 patients (aged 60ะ10 years, 28 male) subjected to coronary surgery between 1992 and 2002. Results: Thirty-four patients presented with unstable angina. On angiography, the LAD had a proximal obstruction in 35 patients. Sixteen presented with a myocardial infarction of the LAD territory. Six were managed previously with angioplasty; four had a new critical obstruction, 1 was catalogued as a procedure failure, and one was totally occluded. There was no operative mortality, myocardial infarction, stroke or need for re operation. There were two late deaths, caused by an advanced cardiac failure at 120 months in one patient, and chronic renal failure at 61 months of follow-up in another. Actuarial survival probability was $100 \%, 93 \%$ and $75 \%$ at 1, 5 and 10 years. Probability of freedom from angina was $98 \%$, and freedom of suffering a new myocardial infarction was $100 \%$ at more than 10 years. The probability of no need for a new coronary procedure (angioplasty or surgery) also was 100\% at more than 10 years. Conclusions: The use of LMA as a coronary bypass graft to LAD is a safe surgical technique, with an excellent duration and permeability and also provides a prolonged time free from cardiac events as mortality, angina, myocardial infarction, and the need of a new coronary procedure (Rev Méd Chile 2005; 133: 881-6).

(Key Words: Angina, unstable; Coronary artery bypass; Mammary arteries; Myocardial revascularization)

Recibido el 2 de noviembre, 2004. Aceptado el 13 de abril, 2005.

Cirugía Cardiovascular, Hospital Regional de Temuco. Departamento de Cirugía, Facultad de Medicina, Universidad de La Frontera. Temuco, Chile.

Correspondencia a: Dr. Juan Carlos Bahamondes S. Departamento de Cinugía, Universidad de La Frontera. Av. Manuel Montt 112, 4ํㅜ piso, Temuco. Fono-Fax: 45-325760. E mail: jcbahamo@ufro.cl 
T as técnicas en cirugía coronaria han continuaLdo evolucionando desde los trabajos pioneros de Sabiston, Favaloro, Green, Spencer y Koles$\operatorname{sov}^{1}$, donde la utilización de la arteria mamaria interna izquierda juega un rol preponderante en la revascularización de la arteria descendente anterior, debido a sus características de permeabilidad mantenida en el largo plazo, al tener menor incidencia de desarrollar ateroesclerosis e hiperplasia intimal. Loop y cols $^{2}$ de Cleveland Clinic, demostraron su excelente permeabilidad a largo plazo (95\% a 10 años), lo cual se traduce en una mejoría sustancial en los resultados clínicos, mejor supervivencia a 10 años, menores reoperaciones y menor cantidad de eventos cardíacos tardíos, en pacientes con lesión de la arteria descendente anterior en quienes se utilizó la arteria mamaria interna izquierda.

En los últimos años, se han desarrollado nuevas técnicas percutáneas, con utilización de catéteres para el tratamiento de lesiones únicas de la arteria descendente anterior y stents intracoronarios, con resultados que demuestran una incidencia considerable de reestenosis. Por otro lado, la cirugía coronaria también ha ido evolucionando en los últimos años, con nuevas técnicas de cirugía sin circulación extracorpórea y el uso de distintos estabilizadores cardíacos, además de abordajes mínimos con distintos resultados ${ }^{3-5}$.

En el presente estudio se establecen los resultados obtenidos en el largo plazo de la revascularización miocárdica con arteria mamaria interna izquierda, en lesiones exclusivas de la arteria descendente anterior.

\section{MATERIAL Y MÉTODO}

Estudio descriptivo de 40 pacientes coronarios con lesión única, tanto en el tercio proximal como en el tercio medio de la arteria descendente anterior, intervenidos en forma consecutiva en el período comprendido entre mayo de 1992 y agosto de 2002 por cirugía cardiovascular del Hospital Regional de Temuco. Se excluyeron a los pacientes con patologías cardiovasculares concomitantes. Previo a la cirugía, a todos los pacientes se le solicitó firmar la solicitud de consentimiento informado.

Los datos fueron obtenidos de fichas clínicas, base de datos de Cirugía Cardiovascular y de protocolos operatorios. El seguimiento a largo plazo se realizó mediante controles clínicos en policlínico de cirugía cardíaca o de las consultas de médicos tratantes, contactos telefónicos; además, se objetivó fecha y causa de muerte en el registro civil.

Se evaluó características angiográficas preoperatorias, infarto miocárdico reciente (definido como aquel menor a 15 días de evolución) y procedimientos de revascularización utilizados previo a la cirugía y las causas de falla en éstos. Se analizó las principales complicaciones postoperatorias ocurridas como infarto miocárdico, accidente cerebrovascular, infección de herida esternal y sangrado postoperatorio que requirió de exploración quirúrgica. En el seguimiento se determinó fecha y causa de muerte alejada, reaparición de angina, infarto miocárdico, y la necesidad de una nueva angioplastia o cirugía de revascularización miocándica.

Técnica quirúrgica: Durante el mismo período, 980 pacientes fueron sometidos a cirugía de revascularización miocárdica exclusiva, correspondiendo esta serie a $4 \%$ del total de operaciones. En todos los pacientes se accedió al corazón mediante esternotomía media clásica, debido a la mejor exposición del campo operatorio y por permitir una disección completa y segura de la arteria mamaria interna izquierda.

Todos los pacientes fueron intervenidos con circulación extracorpórea, cardioplegia cristalina anterógrada, hipotermia moderada a $28^{\circ} \mathrm{C}-32^{\circ} \mathrm{C}$ y magnificación óptica de 2,5-3,0 x.

Cirugía de urgencia: Dos pacientes se operaron de urgencia, por disección del tronco de la coronaria izquierda, producida durante el procedimiento de angioplastia y otro paciente por presentar angina inestable con una lesión hipercrítica proximal de la arteria descendente anterior.

Estadística: Se realizó una estadística descriptiva de la serie y se utilizó el método de Kaplan-Meier para el cálculo de supervivencia actuarial, nuevo infarto miocárdico y necesidad de un nuevo procedimiento de revascularización con angioplastia o cirugía coronaria con el programa computacional Stata $8.0^{\circledR}$. 


\section{RESUlTADOs}

La edad promedio en la serie fue de 60,4 años (DE \pm 9.575, rango 35-77 años), con predominio del sexo masculino en 28 pacientes (70\%), 35 pacientes tenían obstrucción u oclusión proximal de la arteria descendente anterior y 5 pacientes tenían obstrucción del tercio medio. Siete pacientes se presentaron con infarto miocárdico reciente, 6 pacientes con angioplastia previa (Tablas 1 y 2).

Complicaciones angioplastia previa: De los 6 pacientes en que se realizó una angioplastia como tratamiento primario de la lesión, y que posteriormente fueron intervenidos, en 4 se evidenció mediante angiografía reestenosis post angioplastia, en 1 paciente se objetivó oclusión de la arteria en el sitio de la angioplastia; y el procedimiento fue definido como fallido en 1 paciente. A pesar de que en 3 de estos pacientes se había utilizado un stent intracoronario, se evidenció falla del procedimiento a menos de 6 meses post angioplastia.

En relación a las complicaciones derivadas directamente del procedimiento de angioplastia, 1 paciente presentó disección del tronco de la coronaria izquierda siendo intervenido de ungencia, y otro paciente presentó un infarto miocárdico posterior a la angioplastia (Tabla 3).

Morbilidad y mortalidad: De los resultados obtenidos en esta serie no hubo mortalidad, ningún paciente presentó infarto miocárdico perioperatorio, ni tampoco accidente cerebrovascular. No hubo sangrado en el postoperatorio que requiriera reoperación. Un paciente tuvo infección superficial de la herida esternal cuyo cultivo demostró un Staphylococo aureus multisensible, el cual fue tratado con curaciones locales y antibióticos (Tabla 4).

Seguimiento clínico: Se obtuvo 100\% de seguimiento, con un promedio de 43,2 meses (DE \pm 36,58; entre 1-122 meses). Hubo 2 muertes alejadas por: insuficiencia cardíaca avanzada a 120 meses de seguimiento en un paciente con antecedente de un infarto extenso de pared anterior, previo a la cirugía de revascularización y que desarrolló un aneurisma de ventrículo izquierdo; y otro paciente falleció debido a insuficiencia renal crónica avanzada en fase terminal a 61 meses de seguimiento.
Tabla 1. Características clínicas preoperatorias

\begin{tabular}{|lll|}
\hline Características clínicas & $\mathrm{n}$ & $\%$ \\
\hline Hipertensión arterial & 36 & 90 \\
Angor inestable & 34 & 85 \\
Dislipidemia & 20 & 50 \\
Infarto miocárdico previo & 16 & 40 \\
Tabaquismo crónico & 11 & 27,5 \\
Diabetes mellitus & 10 & 25 \\
\hline
\end{tabular}

Tabla 2. C aracterísticas angiográficas preoperatorias

\begin{tabular}{|lrl|}
\hline Características angiográficas & $\mathrm{n}$ & $\%$ \\
\hline Obstrucción ADA* proximal & 35 & 87,5 \\
Obstrucción ADA* tercio medio & 5 & 12,5 \\
Infarto miocárdico reciente & 6 & 15 \\
Angioplastia previa & 6 & 15 \\
\hline
\end{tabular}

${ }^{*}$ Arteria descendente anterior.

\section{Tabla 3. Complicaciones derivadas de la angioplastia previa}

\begin{tabular}{|lcc|}
\hline Complicaciones angioplastia & $\mathrm{n}$ & $\%$ \\
\hline Reestenosis & 4 & 10 \\
Oclusión ADA $^{*}$ & 1 & 2,5 \\
Angioplastia fallida & 1 & 2,5 \\
Stent intracoronario & 3 & 7,5 \\
Disección tronco coronaria izquierda & 1 & 2,5 \\
Infarto miocárdico & 1 & 2,5 \\
\hline
\end{tabular}

${ }^{*}$ Arteria descendente anterior.

\section{Tabla 4. Resultados de la cirugía con arteria mamaria interna}

\begin{tabular}{|lll|}
\hline Resultados & $\mathrm{n}$ & $\%$ \\
\hline Mortalidad & 0 & 0 \\
Infarto miocárdico postoperatorio & 0 & 0 \\
Accidente cerebrovascular & 0 & 0 \\
Reoperación & 0 & 0 \\
Infección superficial esternotomía & 1 & 2,5 \\
\hline
\end{tabular}


En esta serie ningún paciente presentó un nuevo infarto miocárdico posterior a la cirugía, tampoco hubo necesidad de efectuar algún nuevo procedimiento de revascularización con angioplastia o con otra cirugía coronaria. En un paciente hubo reaparición de angina a 120 meses de la operación, siendo estudiado con angiocoronariografía, la que demostró permeabilidad del puente mamario y una nueva lesión no significativa en el territorio de la arteria descendente posterior que fue tratado médicamente. En los 38 pacientes seguidos hasta 150 meses, la sobrevida actuarial global a 1, 5 y 10 años fue de $100 \%$, 93\% y $75 \%$, respectivamente, con un nivel de significación estadística de 95\% (Figura 1).

La probabilidad de estar libre de angina fue de $98 \%$ a más de 10 años (Figura 2). La probabilidad de no requerir un nuevo procedimiento de revascularización (angioplastia o cirugía) fue de $100 \%$ a más de 10 años, como asimismo la probabilidad de estar libre de un nuevo infarto miocárdico es de $100 \%$ en el tiempo de seguimiento observado (Figura 3).

\section{DisCUSIÓN}

Los estudios que han evaluado la historia natural de pacientes con enfermedad coronaria de un vaso, demuestran que el tratamiento médico solo, se asocia con un buen pronóstico a largo plazo, excepto en lesiones severas que comprometen la arteria descendente anterior proximal. Por otro lado, un estudio observacional ${ }^{6}$ sugiere que la angioplastia podría ofrecer una leve superioridad en la sobrevida a largo plazo sobre la cirugía coronaria en pacientes con enfermedad de un vaso, excepto en pacientes con lesiones críticas de la arteria descendente anterior. La angioplastia ha demostrado ser superior al tratamiento médico en términos de mejorar la tolerancia al ejercicio y disminuir la isquemia provocable ${ }^{7-9}$; pero dentro de sus limitaciones se incluye 30 a

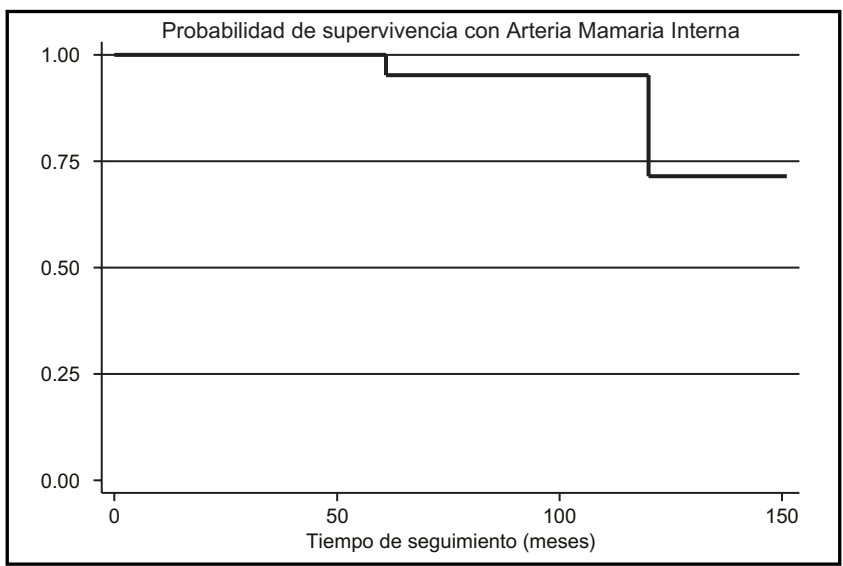

Figura 1. Probabilidad de sobrevida actuarial en pacientes con arteria mamaria interna.

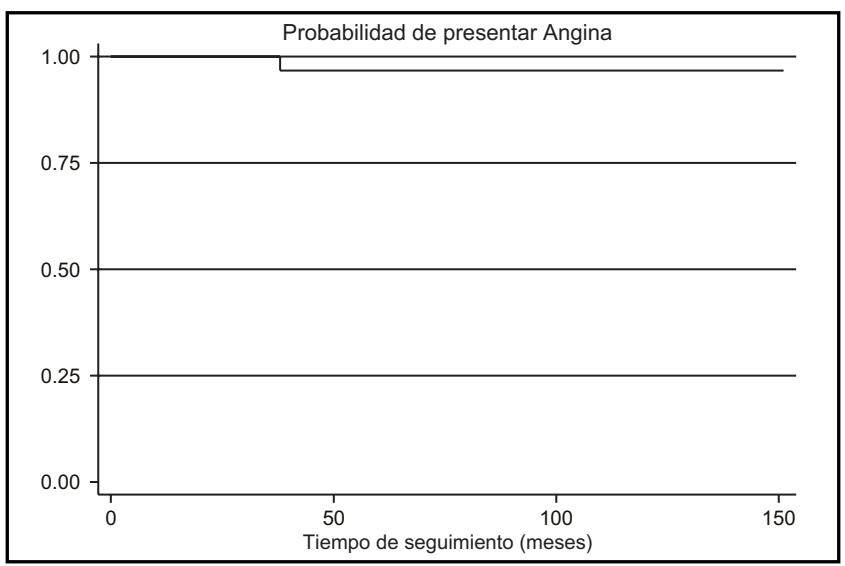

Figura 2. Probabilidad de estar libre de angina en el seguimiento.

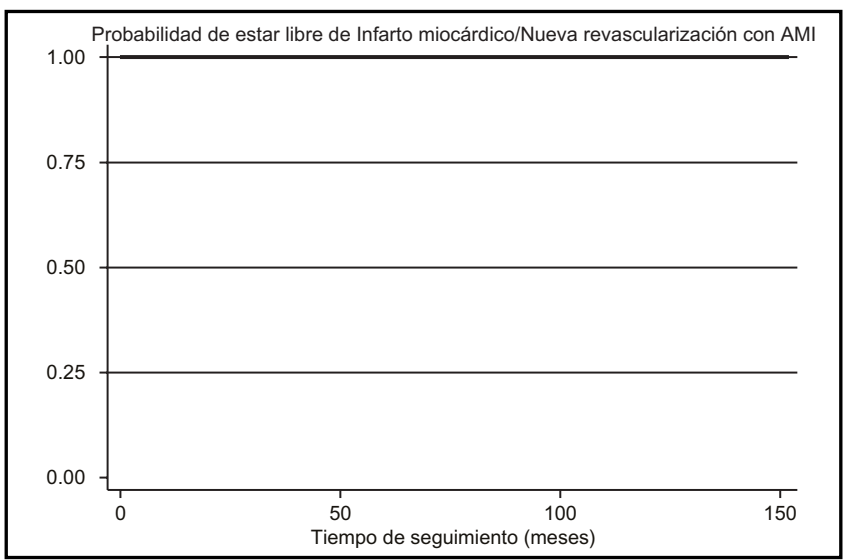

Figura 3. Probabilidad de necesitar un nuevo procedimiento de revascularización/tiempo libre de infarto miocárdico en pacientes con arteria mamaria interna. 
$50 \%$ de reestenosis angiográfica dentro de 3 a 6 meses post procedimiento. La reestenosis se puede presentar como un síndrome isquémico recurrente en aproximadamente $20 \%$ de los pacientes, manifestado por un test de esfuerzo positivo o un patrón de angina in crescendo, lo cual significa la necesidad de un nuevo procedimiento de revascularización en el corto plazo lo cual, en términos económicos, significa a Estados Unidos un total de 2 billones de dólares anuales. Aunque el advenimiento del stent intracoronario ha disminuido la necesidad de una nueva revascularización dentro de 6 meses, la reestenosis intrastent se ha establecido como un problema formidable y de alto impacto económico ${ }^{7,9}$.

La arteria descendente anterior proximal ha demostrado ser el sitio coronario de mayor reestenosis, presentándose angiográficamente en 40 a $50 \%$ después de angioplastia ${ }^{9}$. Un número variado de estudios han evaluado los resultados de la angioplastia versus la cirugía coronaria convencional en estenosis proximal de la arteria descendente anterior. Goy et $\mathrm{al}^{10}$ comparó angioplastia y cirugía con arteria mamaria interna izquierda en 134 pacientes con enfermedad aislada proximal y buena función ventricular, obteniendo $2 \%$ de incidencia de infarto perioperatorio en el grupo quirúrgico y $3 \%$ de incidencia de oclusión aguda del vaso con infarto miocárdico y necesidad de cirugía de revascularización urgente en el grupo de angioplastia. Después de 2 años de seguimiento, el porcentaje de infarto miocárdico fue más alto en el grupo con angioplastia en comparación a la cirugía $(12 \%$ versus $3 \%, \mathrm{p}<0,01)$. No hubo diferencias significativas en cuanto a mortalidad, pero la necesidad de una nueva revascularización fue sustancialmente más alta en el grupo con angioplastia ( $25 \%$ versus $3 \%, \mathrm{p}<0,01$ ), similar a lo encontrado en nuestro estudio. Más aún, a 2 años de seguimiento, un número significativo de pacientes con angioplastia estaba con tratamiento médico antianginoso. Se desprende que, aunque la angioplastia y la cirugía mejoraron el estado clínico, con un riesgo de muerte y de infarto miocárdico similar en pacientes con enfermedad proximal de la arteria descendente anterior, la angioplastia está limitada en sus resultados por un número mayor de reintervenciones. Otros estudios demuestran retrospectivamente resultados y calidad de vida en pacientes en quienes se utilizó la arteria mamaria interna, demostrando en los pacientes una mayor supervivencia libre de angina, infarto miocárdico y necesidad de nueva revascularización ${ }^{11-15}$ como es posible observar en nuestro estudio con 98\% libre de angina, y $100 \%$ libre de infarto y una nueva revascularización a 5 y 10 años. Similares resultados se observó en el estudio prospectivo a 3 años MASS (Medicine, Angioplasty, or Surgery Study) ${ }^{16}$.

En nuestro medio, existen pocas comunicaciones referentes a este problema, destacando el estudio de revascularización miocárdica de la arteria descendente anterior con técnica clásica de la Universidad Católica ${ }^{16}$, con un seguimiento promedio de 64 meses, que muestra una probabilidad de sobrevida y libre de otro procedimiento de revascularización de $100 \%$ a 12 meses, disminuyendo solamente a $96 \%$ y $97 \%$ a 5 años de seguimiento, respectivamente. Nuestros resultados son similares en cuanto a los objetivos planteados, destacando la baja incidencia de angina en el seguimiento y la nula necesidad de realizar una nueva cirugía o procedimiento percutáneo. La probabilidad de sobrevida actuarial observada a 1 año es de $100 \%$ y de $93 \%$ a 5 años, y ésta disminuye a $75 \%$ a 10 años de seguimiento debido a la alta morbilidad de los pacientes previo a su cirugía, como se observa en la Figura 1. Aun así, nuestros resultados son comparables a casuísticas de otros grupos quirúrgicos ${ }^{17-20}$.

Por otra parte, creemos que la combinación de una biología única de la arteria mamaria interna pediculada y el gran flujo sanguíneo diagonal y septal de la arteria descendente anterior, provee de un puente arterial de larga duración, que permite tiempos prolongados de supervivencia libre de eventos como angina, infarto miocárdico, necesidad de angioplastia o cirugía y mortalidad. Como se demuestra en este estudio, la técnica empleada de injerto pediculado de arteria mamaria interna a la arteria descendente anterior, mediante esternotomía media, es una técnica extremadamente segura, sin mortalidad y prácticamente sin morbilidad asociada. 


\section{REFERENCIAS}

1. KolesSov VI. Mammary artery-coronary artery anastomosis as a method of treatment for angina pectoris. J Thorac Cardiovasc Surg 1967; 54: 53544.

2. Loop FD, Lytle BW, Cosgrove DM, Stewart RW, Goormastic M, Wiwams GW. Influence of the internal mammary graft of 10 years survival and other cardiac events. N Engl J Med 1986; 314: 1-6.

3. Benetti FJ, Nasem G, Wood M, GefFner L. Direct myocardial revascularization without extracorporeal circulation. Experience in 700 patients. Chest 1991; 100: 312-6.

4. Buffolo E, De Andrade CS, Branco JN. Comonary artery bypass grafting without cardiopulmonary bypass. Ann Thorac Surg 1996; 61: 63-6.

5. Calafiore AM, Gianmarco G, Teodori G, Gauna $S$, Maddestra N, Paloscia L. Midterm results after minimally invasive coronary surgery (Last operation). J Thorac Cardiovasc Surg 1998; 115: 763-77.

6. Goy JJ, Eeckhout E, Burnand B, Vogt P, Stauffer JC. Coronary angioplasty versus left internal mammary artery grafting for isolated proximal left anterior descending artery stenosis. Lancet 1994; 343: 1449-53.

7. Serryus PW, Strauss BH, Beatt KJ, Bertrand $M$, Puel J, Rickards A et al. Angiographic follow-up after placement of a self expanding coronary artery stent. N Engl J Med 1991; 324: 13-7.

8. Schatz RA, Baim DS, LeON M, Eus S, Goldberg S, HiRschFFeLD J ET AL. Clinical experience with the Palmaz-Schatz coronary stent. Inicial results of a multicenter study. Circulation 1991; 83: 148-60.

9. Versaci F, Gaspordone A, Tomai F, Crea F, Chiarelo L, GiofrRe P. A comparison of coronary artery stenting with angioplasty for isolated stenosis of the proximal left anterior descending coronary artery. N Engl J Med 1997; 336: 817-22.

10. Goy JJ, Eeckhout E, Moret C, Burnand B, Vogt P, StaufFer JC et al. Five years outcome in patients with isolated proximal left anterior descending coronary artery stenosis trated with angioplasty or left internal mammary artery grafting. A prospective trial. Circulation 1999; 99: 3255-9.

11. Morán S, Larraín E, Becker P, Irarrázaval MJ, Urzúa J, LeMA G ET aL. Evolución de las indicaciones, técnica quirúrgica y resultados de la revascularización miocándica. Rev Chil Cardiol 1993; 12: 13-7.
12. Morán S, Irarrázaval MJ, Zalaquett R, Viliavicencio M, Garayar B, MuÑoz C et al. Revascularización miocárdica con arteria mamaria interna bilateral. Rev Chil Cardiol 1996; 15: 4-8.

13. Morán S, Irarrázaval MJ, Zalaquett R, Vimavicencio M, Garayar B, Muñoz C et al. Revascularización miocárdica con una y dos arterias mamarias: resultados clínicos y seguimiento alejado. Rev Méd Chile 1997; 125: 391-401.

14. Irarrázaval MJ, Muñoz C, Garayar B, Morán S, Zalaquett R, Maturana G et al. Cirugía coronaria. Veinte años de seguimiento. Rev Méd Chile 1998; 126: 63-74.

15. Hueb WA, Belotti G, Almeida De Olvveira SA, Arie S, Piva De Albuquerque C, Jatene A et al. The Medicine, Angioplasty or Surgery Study (MASS): a prospective, randomized trial of medical therapy, ballon angioplasty or bypass surgery for single proximal left anterior descending artery stenoses. J Am Coll Cardiol 1995; 26: 1600-5.

16. Garayar B, Irarrázaval MJ, Morán S, Zalaquett R, BeCKer P, Maturana G ET aL. Revascularización miocárdica de la arteria descendente anterior con anastomosis mamaria con técnica clásica. Rev Esp Cardiol 2000; 53: 316-20.

17. Tavila G, Kappetein AP, Braun J, Gopie J, TJien AT, Dion RA. Long-term follow up of coronary artery bypass grafting in the vessel disease using exclusively pedicled bilateral internal thoracic and right gastroepiploic arteries. Ann Thorac Surg 2004; 77: 794-9.

18. Shah PJ, Durairaj M, Gordon I, Fuler J, Rosalion A, Seevanayagam P et al. Factors affecting patency of internal thoracic artery graft: clinical and angiographic study in 1434 symptomatic patients operated between 1982 and 2002. Eur J Cardiothorac Surg 2004; 26: 118-24.

19. Rzzzol G, Schiavon L, Bewni P. Does the use of bilateral internal mammary artery (IMA) graft provide incremental benefit relative to the use of a single IMA graft? A Meta-analysis approach. Eur J Cardiothorac Surg 2002; 22: 781-6.

20. Godman S, Zadina K, Moritz T, Ovitt T, Sethi G, Copeland JG ET AL. Long term patency of saphenous vein and left internal mammary artery grafts after coronary artery bypass surgery: results from the department of Veterans Affairs Cooperative Study. J Am Coll Cardiol 2004; 7; 44: 2149-56. 